\title{
Development of a simple procedure for the treatment of femoral head osteonecrosis with intra-osseous injection of bone marrow mesenchymal stromal cells: study of their biodistribution in the early time points after injection
}

Angélique Lebouvier ${ }^{1,2,3}$, Alexandre Poignard ${ }^{1,3,4}$, Madeleine Cavet ${ }^{5}$, Jérôme Amiaud ${ }^{6}$, Julie Leotot ${ }^{1,2,3}$, Philippe Hernigou ${ }^{1,3,4}$, Alain Rahmouni ${ }^{5}$, Philippe Bierling ${ }^{2,3}$, Pierre Layrolle ${ }^{6}$, Hélène Rouard ${ }^{1,2,3,7 \dagger}$ and Nathalie Chevallier ${ }^{1,2,3^{*}+}$

\begin{abstract}
Introduction: Osteonecrosis of the femoral head (ONFH) is a degenerative disease progressing to a femoral head (FH) collapse. Injection of osteoprogenitor cells like bone marrow mesenchymal stromal cells (BMSCs) into the FH appears to be a good therapeutic treatment. However, safety and efficacy of BMSCs to treat bone defect are the main preclinical data required for clinical application. Efficacy and the lack of risk of cell transformation after amplification of BMSCs have been extensively described. The main objectives of this study were to develop a simple and usable procedure for clinicians and control its feasibility by evaluating the biodistribution of BMSCs after injection into the FH in a large animal model. The impact of this approach was evaluated on one natural pig ONFH.

Methods: BMSCs were directly injected in the pig FH, and then the biodistribution of grafted cells was detected by quantitative real-time polymerase chain reaction, cytometry, or a combination of classic histology analysis and in situ hybridization (ISH). BMSC efficacy on bone regeneration was evaluated by magnetic resonance imaging (MRI) and histology.

Results: After 30-minute and 24-hour follow-up, grafted cells were detected th the injection site and no BMSCs were detected in filter organs or body fluids. The combination of classic histology analysis and ISH showed a good homogeneity of cell distribution in FH. Local delivery of BMSCs onto a bone scaffold associated with bone formation in vivo confirmed the preferential tropism of BMSCs to the bone tissue as well as their efficacy to form bone. Treatment of a natural pig ONFH by autologous BMSCs indicated a beginning of bone healing as early as 2 weeks with a complete healing after 9 weeks. At this stage, MRI and histological analysis were similar to those of a normal FH.
\end{abstract}

Conclusions: Intra-Osseous injection of BMSCs in FH seems to be a good strategy for ONFH treatment as the safety concerning the biodistribution of BMSCs is ensured. Moreover, the efficacy of BMSCs in natural ONFH seems to indicate that this is a promising approach. Altogether, these results constitute the preclinical data necessary for the setup of a clinical application with expanded BMSCs in the context of advanced therapy medicinal products.

\footnotetext{
* Correspondence: nathalie.chevallier@efs.sante.fr

${ }^{\dagger}$ Equal contributors

'Université Paris-Est, Faculté de médecine, Laboratoire de "Bioingénierie cellulaire, tissulaire et sanguine", EA3952, 5 rue Gustave Eiffel, 94000 Créteil, France

${ }^{2}$ Etablissement Français du Sang d'lle-de-France, Unité d'Ingénierie et de Thérapie Cellulaire, 5 rue Gustave Eiffel, 94017 Créteil cedex, France Full list of author information is available at the end of the article
} 


\section{Introduction}

Osteonecrosis of femoral head (ONFH) is a progressive degenerative disease due mainly to the loss or compromise of blood flow to the femoral head (FH) and bone progenitor deficiency. If the necrotic bone lesion is not treated early, it may progress to a collapse of FH and require a total hip replacement [1]. This painful disorder commonly occurs in a young population (mean age of 36 years) [2]. To avoid arthroplasty, many conservative procedures are used in the early pre-collapse stage of ONFH, including core decompression associated (or not) with autologous bone marrow (BM) grafting $[3,4]$. However, even if positive results are obtained, the treatment of the ONFH continues to be a challenging problem for orthopedic surgeons. Bone tissue engineering, using mesenchymal stromal cells (MSCs), provides a promising approach [5]. Indeed, MSCs used in various animal models of bone repair were described to have significant osteogenic potential [6-8], and promising case reports have been published $[9,10]$.

MSCs have the potential to migrate and the capacity to be mobilized to sites of injury. However, it has been shown that injected MSCs via intra-artery and intravenous (IV) portals lead to their detection in the lungs within 15 minutes and then in the liver, kidneys, and spleen, indicating a large spectre of cell dissemination [11-14]. Several articles seem to indicate that if the cells are injected in the site of injury, they stay preferentially and with a better viability to this site compared with an IV injection [13-15]. As bone is the physiological environment of BM-MSCs (BMSCs), we hypothesized that a local delivery of BMSCs into the FH during surgery would facilitate their location and participation in tissue regeneration.

For clinical applications of this advanced therapy medicinal product (ATMP), preclinical data on BMSC safety concerning their innocuity and their biodistribution after their injection are required [16,17]. It has been previously shown that there is no risk of BMSC transformation after their amplification and graft in vivo [18]. Currently, it is necessary to demonstrate the homing pattern of the injected cells to avoid inappropriate differentiation in other organs or the development of cancer cells [18-21]. Cells spreading can be impacted by the route of administration [14]. Therefore, to assess the biodistribution, it is essential to administer the cells by the exact portal that will be used in the clinic. Preclinical recommendations require to perform the test with human BMSCs (hBMSCs) on a large animal model of the disease [17]. The pig is considered a translational model in biomedical research because of anatomical, physiological, and biochemical similarities to humans and has been commonly used to obtain preclinical data [22,23].
The goals of our work were to develop a new therapeutic treatment of ONFH by injecting BMSCs directly into the $\mathrm{FH}$ without using any BMSC carrier and to make the orthopedic surgery procedure easy and suitable for clinicians. One essential piece of preclinical data required for this procedure is the analysis of their biodistribution in a large animal model. To this end, human or pig BMSCs were directly injected in the pig $\mathrm{FH}$ as is done in the clinic for the injection of the concentrated $\mathrm{BM}$ with a trocar of $4 \mathrm{~mm}$ in diameter. To check whether grafted BMSCs are confined to the target site and not found in proximal tissues or filter organs such as lungs and kidneys or body fluids (blood and BM), we used different highly sensitive techniques like cell cytometry analysis and an innovative approach using species-specific human primers for quantitative realtime polymerase chain reaction (qPCR). In parallel, to localize the human cells, we conducted classic histology analysis associated with in situ hybridization (ISH) of the human Alu sequence. To confirm the preferential tropism of BMSCs to bone, a local delivery of BMSCs onto a bone scaffold in a mouse model was performed in vivo and BMSC efficacy was also evaluated on one natural pig ONFH.

\section{Methods}

\section{Animals}

Ethical approval for all animal experimentation was obtained from the local ethics committee (ComEth Afssa/ ENVA/UPEC, Maisons-Alfort, France) (\#12-036) in accordance with the European Guidelines for Animal Care (Directive 2010/63/EU).

\section{Pigs}

Five female pigs (hybrid of Landrace and large white pigs) with a weight of 35 to $50 \mathrm{~kg}$ and age of 3 to 6 months were used (Lebeau Christian, Gambais, France). Pigs were managed in accordance with the instructions of the ethics committee.

\section{Mice}

Two severe combined immunodeficiency (SCID) mice (males, 7 weeks old) purchased from Charles River Laboratories (Chatillon, France) were used for the ectopic implantation procedure. The mice were anesthetized with isoflurane (Abbott, Rungis, France) and were euthanized with an overdose of pentobarbital (Centravet, Maisons-Alfort, France).

\section{Biomaterials}

Scaffolds of Tutoplast Process Bone (Tutogen Medical, Metz, France) were derived from human cancellous bone. The Tutoplast process consisted of a delipidization, an osmotic cell destruction treatment, hydrogene 
peroxide treatment, and washing cycles for removal of the non-collagen proteins followed by a solvant dehydrated step and finally a $\gamma$-irradiation procedure. Fragments of 2 to $4 \mathrm{~mm}$ were cut manually and were stored at room temperature (RT) under sterile condition. Bone scaffolds of equivalent size, volume, and weight $(8.0 \pm$ $1.0 \mathrm{mg}$ ) were used in this study to ensure a comparable surface area for in vivo analyses.

\section{Bone marrow mesenchymal stromal cell cultures}

Pig BMSCs were isolated from BM (5 to $10 \mathrm{~mL}$ ) of pig humerus (pBMSCs). Human MSCs were isolated from BM (3 to $5 \mathrm{~mL}$ ) collected from the iliac crest (hBMSCs) of patients undergoing standard $\mathrm{BM}$ transplantation procedures (AP-HP Hôpital Henri Mondor, Créteil, France), after having received their informed consent in accordance with the Declaration of Helsinki. The project was approved by the Ethical Committee of Ile de France (section 4 \#DC-2009-1049). pBMSCs and hBMSCs were cultured in alpha-modified Eagle's medium ( $\alpha M E M$ ) (PAA, Les Mureaux, France) supplemented with 10\% of foetal calf serum (FCS) (Stem Cell Technologies, Grenoble, France) and 0.5\% ciprofloxacine (Bayer Pharma, Puteaux, France). The hBMSCs used in this study were positive for CD90, CD105, and CD73 and negative for CD34 and CD45 and were able to differentiate into osteogenic, adipogenic, and chondrogenic lineages (data not shown) as previously described $[7,24,25]$.

\section{Functional characterization}

To characterize pBMSCs, their capacity to differentiate into mesenchymal lineages was assessed. For osteogenic differentiation, at $50 \%$ confluence the growth medium was replaced by $\alpha M E M-10 \%$ FCS supplemented with $50 \mu \mathrm{M}$ L-ascorbic acid-2-phosphate (AA), $10 \mathrm{mM}$ ßGlycerophosphate ( $\beta$ Gly), $0.1 \mu \mathrm{M}$ dexamethasone (Dex) (Sigma, Saint Quentin Fallavier, France), and 100 ng/mL $\mathrm{rhBMP}_{2}$ (recombinant human bone morphogenetic protein 2, Inductos; Laboratoire Wyeth Pharmaceuticals, Philadelphia, PA, USA). On day 10, the monolayers were fixed in $70 \%$ ethanol (Cooper, Melun, France) for 1 hour at $4^{\circ} \mathrm{C}$ and stained for 15 minutes with Alizarin Red $\mathrm{S}$ (Sigma) at RT.

For adipogenic differentiation, at $80 \%$ confluence the medium was replaced by a high-glucose medium (Invitrogen, which is part of Life Technologies, Villebon sur Yvette, France) supplemented with 10\% FCS, $0.1 \mathrm{mM}$ Dex, $0.2 \mathrm{mM}$ indomethacin, $0.01 \mathrm{mg} / \mathrm{mL}$ insulin, and 0.5 mM IBMX (Sigma). On day 10, the monolayers were fixed by using $4 \%$ paraformaldehyde (VWR, Fontenay Sous Bois, France) for 5 minutes at RT and stained for 15 minutes with $0.3 \%$ Oil Red O (Sigma)/60\% isopropanol (VWR). Chondrogenic differentiation was performed in pellet culture by using Stempro, a Chondrogenesis
Differentiation Kit (Life Technologies), as described by the manufacturer. On day 21, pellets were fixed in $4 \%$ formaldehyde (Sigma) and embedded in paraffin. Sections $(3 \mu \mathrm{m})$ were stained with Alcian Blue 8GX (Sigma) as described by the manufacturer and counterstained with hematoxylin (Sigma).

\section{Surgical procedure in pigs}

Pigs were managed in accordance with the instructions of the ethics committee. Access to FH was done in accordance with the previously described protocol by a percutaneous approach of the hip [26]. Pigs received an injection of $140 \times 10^{6}$ autologous pBMSCs $(n=1)$ or hBMSCs $(\mathrm{n}=2)$ in $7 \mathrm{~mL}$ of $5 \%$ human serum albumin (Albunorm; Octapharma, Boulogne-Billancourt, France), and one pig served as a negative control. To push all the cells inside the FH and to allow the cells to migrate correctly to the necrotic site, a volume of air (2 to $5 \mathrm{~mL}$ ) was injected and then the trocar was let 5 minutes before being removed.

Blood was collected before (T0) and after injection with a kinetic from 1 minute to 24 hours. Liver, kidneys, spleen, and lungs were collected at either 30 minutes or 24 hours after injection. BM was collected before (T0) and 24 hours after injection. Injected $\mathrm{FH}$ and adjacent tissues (that is, capsule, periarticular muscles, gluteus maximus muscle, and round ligament) were analysed 30 minutes after injection. Non-injected pig served as a negative control. Organs were dissected into several pieces and ground on a cell strainer with a suitable piston and used for cytometry and molecular biology.

\section{Cell labeling with $\mathrm{DiOC}_{18}$}

Before injection, pBMSCs $\left(20 \times 10^{6}\right.$ cells $\left./ \mathrm{mL}\right)$ were incubated in $10 \mu \mathrm{g} / \mathrm{mL}$ of $\mathrm{DiOC}_{18}$ (3, 3'-dioctadecyloxacarbocyanine perchlorate) solution (Molecular Probes, part of Life Technologies) containing 3\% FCS for 20 minutes at $37^{\circ} \mathrm{C}$ [27]. Finally, pBMSCs were washed with $1 \mathrm{X}$ Hanks' balanced salt solution (PAA) three times to get rid of dye remnant and re-suspended in 5\% human serum albumin (Albunorm; Octapharma).

\section{Flow cytometry}

Ground organs and body fluids of pigs that received an hBMSC injection were stained for BMSC marker CD73APC (Becton, Dickinson and Company, Franklin Lakes, NJ, USA) for 15 minutes. Then the different samples of pigs that received an injection of $\mathrm{DiOC}_{18}^{+}$pBMSCs $(\mathrm{n}=1)$ or hBMSCs $(n=2)$ were examined by using FACSCanto ${ }^{\text {ma }}$ II (Becton, Dickinson and Company). The data were analysed by using BD FACS DIVA ${ }^{\mathrm{mm}}$ software (Becton, Dickinson and Company). The efficacy of labeling was checked before injection with positive expression of stained cells defined as fluorescence greater than $95 \%$ of 
that of the corresponding control pBMSCs not labeled $\mathrm{DiOC}_{18}$ or hBMSCs not labeled CD73.

\section{DNA purification and quantitative real-time polymerase chain reaction}

Two specimens were sacrificed 30 minutes and 24 hours after hBMSC injection. Samples were immediately placed in DNA lysis buffer (Qiagen, Courtaboeuf, France) after collection. Total DNA was isolated by using a QIAmp DNA Mini Kit for blood, BM, organs, and tissue samples and using a QIAmp DNA Investigator Kit for FH samples as described by the manufacturer (Qiagen). FH samples were previously pulverized to a fine powder by using a ceramic ball of $6.35 \mathrm{~mm}$ and a Fast Prep System (MP Biomedical, Santa Ana, CA, USA). The human genomic DNA (gDNA) obtained was quantified by using human TaqMan Copy Number Reference Assay, RNase P (Applied Biosystems, part of Life Technologies, Courtaboeuf, France) with a 7500HT Fast Real-Time PCR System (Applied Biosystems). hBMSC standard range was realized with decreasing concentrations of hBMSC gDNA diluted in pig gDNA $(5 \mathrm{ng} / \mu \mathrm{L})$. The straight equation $\left(y=-3.526 \times 38.163 ; R^{2}=0.9983\right)$ of standard curve had permitted us to obtain the number of cells corresponding to detected cycle threshold $(\mathrm{Ct})$.

\section{In situ hybridization of human Alu sequences}

After euthanasia, half of $\mathrm{FH}$ was removed 30 minutes after hBMSC injection, fixed for 48 hours, and decalcified in 4.13\% EDTA solution (pH 7.4) (Sigma). After dehydration, clearing, paraffin-embedding, and cutting steps, ISH was performed on the FH sections as previously described by Redwine and Armstrong [28]. Locked nucleic acid-based probes were ordered from Exiqon, Inc. (Woburn, MA, USA), and the sequence used was /5DigN/TCTCGATCTTCCTGACCTCATGA/3Dig_N/. Sections were deparaffinized, rehydrated, washed, and treated with $3 \%$ hydrogen peroxide for 15 minutes. After washing, sections were treated in $0.1 \mathrm{M}$ triethanolamine $\mathrm{pH} 8.0$ and $0.25 \%$ acetic acid for 20 minutes at RT and pre-hybridized for 1 hour at $56^{\circ} \mathrm{C}$ in buffer containing 4X SSC (sodium saline citrate) (VWR), 50\% deionized formamide, 1X Denhardt's solution, 5\% Dextrane Sulfate, and $100 \mu \mathrm{g} / \mathrm{mL}$ Salmon Sperm DNA. Hybridization buffer was replaced by fresh buffer containing $70 \mathrm{nM}$ of Alu probe and was denatured for 5 minutes at $95^{\circ} \mathrm{C}$. Hybridization was carried out for 2 hours at $56^{\circ} \mathrm{C}$ in a wet chamber. Slides were washed twice for 5 minutes in 2X SSC and twice for 5 minutes in $0.5 \mathrm{X} \mathrm{SSC}$ at $56^{\circ} \mathrm{C}$ each. Signals were detected by using anti-DIG horseradish peroxidase-conjugated Fab fragments (Roche, Boulogne Billancourt, France) and diaminobenzidine (Dako, Carpinteria, CA, USA) as substrate. Sections were counterstained with Gill-2 hematoxylin (Thermo Shandon
Ltd., Runcorn, UK). Two negative controls were produced to compare and ensure consistent interpretation: negative controls of pig FH injected with human cells in omitting Alu probe and sections of $\mathrm{FH}$ injected with physiological saline only exposed to Alu probe. Slides were observed by using a DMRXA microscope (Leica, Nussloch, Germany).

\section{Magnetic resonance imaging}

Magnetic resonance imaging (MRI) was performed on a 1.5-T MRI device (Siemens Avanto, Erangen, Germany). Pigs were in the supine position, and their hind legs were tied in extension. T1-weighted and T2 with fat saturation (T2 FS)-weighted sequences were obtained in the coronal planes.

Ectopic implantation procedure in immunodeficient mice Six subcutaneous dorsal pockets $(0.5-\mathrm{cm}$ incisions) were prepared in each of the SCID mice. In each pocket, one scaffold was implanted and 300,000 pBMSCs (P2) were injected onto the scaffold in the pocket. The skin was closed with 5-0 sutures (Ethicon, San Lorenzo, Puerto Rico, USA). Cell-free scaffolds were implanted under similar conditions and served as controls. pBMSCs from porcine $\mathrm{BM}$ of three independent pigs were tested in duplicate ( $\mathrm{n}=6$ scaffolds).

\section{Histology \\ Pig femoral head}

Pig FHs were fixed with $4 \%$ formaldehyde solution (VWR), decalcified in $6.8 \%$ nitric acid (VWR) for 2 weeks, and rinsed abundantly in tap water before embedding in paraffin. Sections $(3 \mu \mathrm{m})$ were stained with Masson's Tri-chrome (hematoxylin: nuclear staining; acid fuchsin/xylidine ponceau: cytoplasmic staining; light green SF yellowish: collagen staining; all from VWR). Images were visualized by standard light microscopy and captured by using a UC30 Digital Color Camera and CellSens Entry software (Olympus, Rungis, France).

\section{Mice scaffolds}

After 7 weeks, scaffolds were excised from mice and immediately fixed in $70 \%$ ethanol, decalcified for 3 hours in $6.8 \%$ nitric acid (VWR), and rinsed in tap water before embedding in paraffin. Sections (3 to $5 \mu \mathrm{m}$ ) were stained with Masson's Tri-chrome. Fifteen sections of each sample were analyzed (five at the beginning, five in the middle, and five at the end).

\section{Results}

Having previously described that only $7 \mathrm{~mL}$ can be injected in the FH (data not shown) [29] and published that a concentration of $20 \times 10^{6} \mathrm{BMSCs} / \mathrm{mL}$ is efficient to induce bone formation $[7,8,24]$, we decided for the future clinical protocol to inject $140 \times 10^{6}$ BMSCs in the 
FH. To access cell diffusion after injection in the pig FH, three different protocols were performed: two with human cell origins (hBMSCs) with a 30-minute or 24-hour follow-up and one with autologous pig cells (pBMSCs) with a 24-hour follow-up. After injection, the cells were monitored by flow cytometry, molecular biology, or histology, at different times and in various organs.

\section{Biodistribution of human bone marrow mesenchymal stromal cells after intra-osseous injection in pig femoral head}

Preclinical recommendation required us to assess the biodistribution with human cells [17], and hBMSCs were injected in the pig FH. Compared with unlabeled hBMSCs (dark histogram), the human cells stained for hCD73 were all positive for this marker (grey histogram) (Figure 1). Collected blood at 0 (before injection), 1, 5, 15, and 30 minutes and 1 hour and 24 hours post-injection and collected BM at 0 minutes and 24 hours post-injection were analysed by flow cytometry (Figure 1A, C). Compared with the control blood and control BM (white histogram), no positive cells for hCD73 (grey histogram) were found. Twenty-four hours after injection, no hCD73-positive cells were detected in the liver, lungs, spleen, and kidneys or in adjacent tissues such as periarticular muscles and round ligament (Figure 1B). Only normal tissue-specific cells were found (white histogram).

To confirm the cytometry study, an approach by species-specific qPCR was developed. To quantify the presence of human cells injected in the porcine $\mathrm{FH}$, a
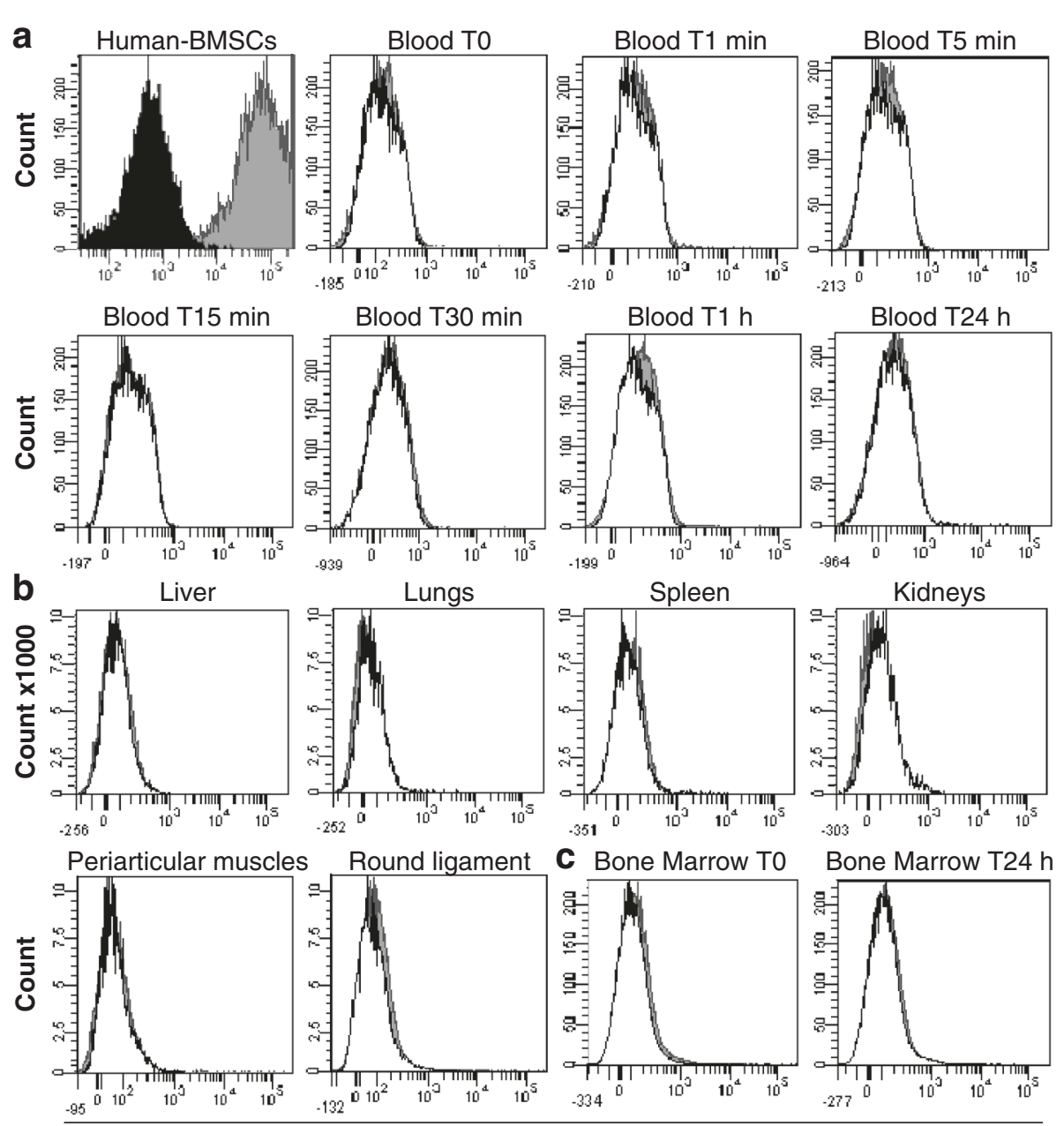

C Bone Marrow T0

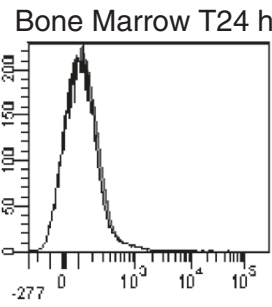

hCD73-APC

Figure 1 Biodistribution analysis of injected human bone marrow mesenchymal stromal cells (hBMSCs) in pig femoral head by flow cytometry. hBMSCs were injected in the subchondral area of pig femoral head and analysed at different time point. (a) Blood was collected before (T0) and after injection with a kinetic of 1 minute to 24 hours. (b) Liver, lungs, spleen, and kidneys were analysed either at 30 minutes or 24 hours after injection. Periarticular muscles and round ligament were collected 30 minutes after injection. (c) Bone marrow was collected before (T0) and 24 hours after injection. Dark histogram: unstained hBMSCs; grey histogram: stained CD73-APC hBMSCs; and white histogram: negative control cells of a non-injected pig. 

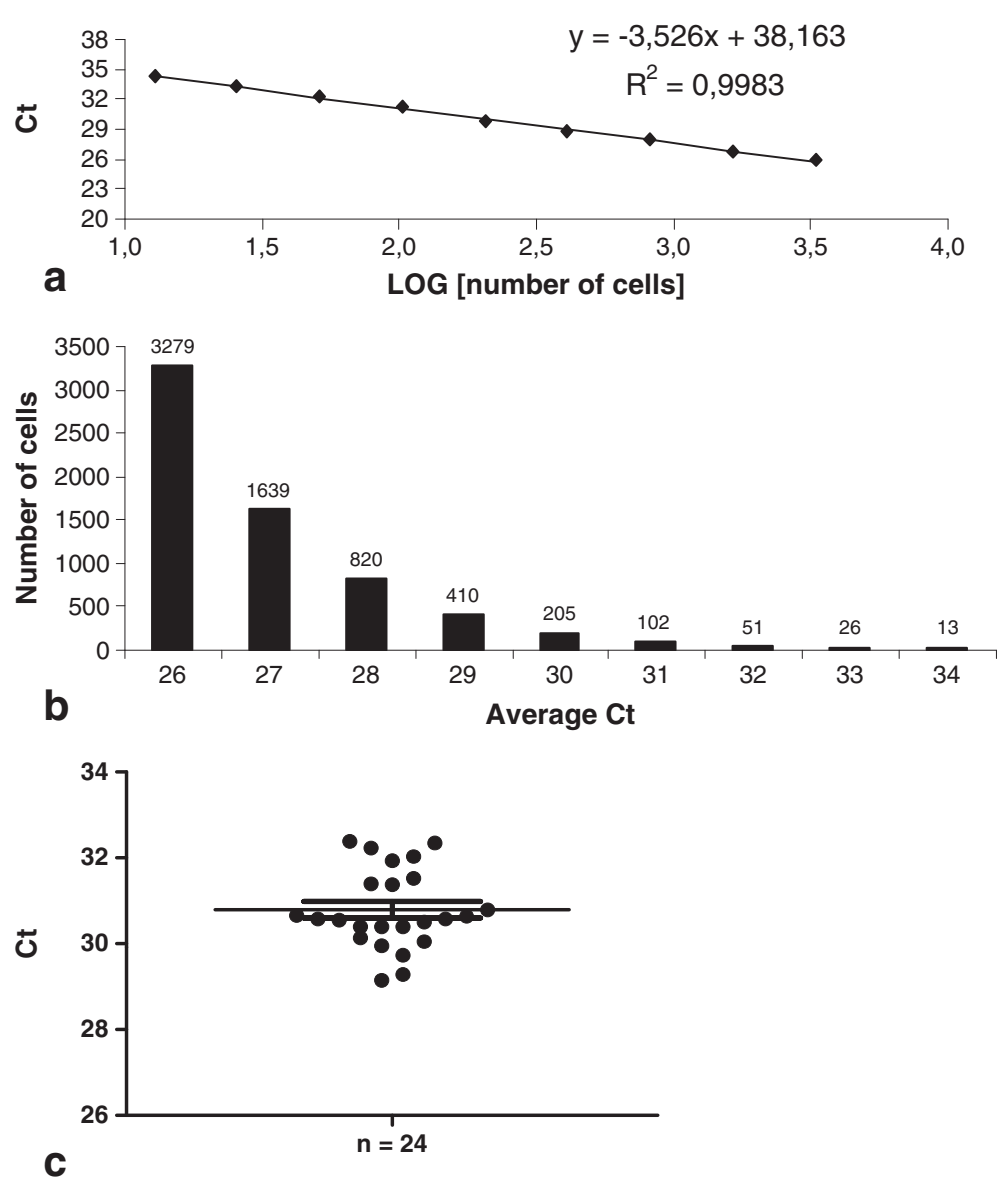

Figure 2 Tracking of injected human cells in pig femoral head by molecular biology. (a) Standard curve of human cells quantified by using human TaqMan Copy Number Reference Assay, RNase P. (b) Conversion of average cycle threshold (Ct) to corresponding number of cells from the straight equation of the standard curve $(y=-3.526 \times 38.163)$. (c) Detection $(C t)$ of injected cells in pig femoral head samples $(n=24)$ by reverse transcription-quantitative real-time polymerase chain reaction with human RNase $P$.

standard curve was established. For this purpose, human gDNA was serially diluted in pig DNA. The linear curve obtained indicated that our qPCR primer (RNase P) was $100 \%$ efficient $\left(R^{2}=0.99\right)$ (Figure 2a). This curve allowed us to quantify the exact number of cells up to 13 human cells when mixed in 3,200 pig cells. With this standard curve, we determined our sensitivity threshold at $0.41 \%$ (Figure 2B). Below 34 cycles, the standard curve was not linear; human cells were detected but were not quantifiable. Two independent assays were performed: one with a 30-minute follow-up and the other with a 24-hour follow-up. In both cases, no human cells were found in adjacent tissues of $\mathrm{FH}$ ( $\mathrm{n}=6$ samples per tissue) or in blood, lungs, liver, spleen, and kidneys $(\mathrm{n}=4$ samples for blood and $n=6$ samples per organ). BM of the noninjected pig humerus was also tested, and as in the other organs, no human cells were found $(n=4)$ (Table 1$)$. As expected, 30 minutes after human cell injection, the injected hBMSCs were found in the FH. All FH samples tested by RNase P qPCR were positive, with a cycle threshold (Ct) average of $30.8 \pm 0.9$, which indicated an average of $147 \pm 21$ cells per $10 \mathrm{mg}$ of $\mathrm{FH}$ samples $(\mathrm{n}=$ 24) (Figure 2c).

Histological analysis was performed to localize the injected cells in the FH. Normal FH showed trabecular bones surrounded by BM endowed with hematopoietic cells and adipocytes (Figure 3a, b). After hBMSCs injection, part of the $\mathrm{BM}$ was replaced by large nonhematopoietic cells (arrows). These cells were localized close to trabeculae and seemed to be attached to the bone surface (Figure 3c, d). To confirm histologic findings, a human-specific Alu probe was used for Alu-ISH (Figure 3e-i). Positive brown nuclei staining was considered positive for Alu-ISH. Negative controls did not show any brown cytoplasmic or nuclear staining (Figure $3 \mathrm{f}, \mathrm{g}$ ). The sections of FH with injected human cells showed positive dark brown nucleus staining, whereas other cells were stained only with hematoxylin (Figure $3 \mathrm{~h}, \mathrm{i})$. Human cells were well distributed inside the pig $\mathrm{FH}$ but were localized in the FH subchondral area on either side of the 
Table 1 Tracking of injected human bone marrow mesenchymal stromal cells in vivo by molecular biology: detection by quantitative real-time polymerase chain reaction of the human RNase $P$ gene

\begin{tabular}{|c|c|c|c|c|}
\hline \multicolumn{3}{|l|}{ Samples } & \multirow{2}{*}{$\begin{array}{l}\text { Delay after injection } \\
\text { No injection }\end{array}$} & \multirow{2}{*}{$\begin{array}{l}\text { RNase detection } \\
\text { No detection }\end{array}$} \\
\hline Pig negative control & Organs & Lung & & \\
\hline & & Liver & & \\
\hline & & Spleen & & \\
\hline & & Kidney & & \\
\hline & Blood & & & \\
\hline & Bone marrow & & & \\
\hline \multirow[t]{17}{*}{ Pig sample after hBMSCs injection } & \multirow{4}{*}{$\begin{array}{l}\text { Adjacent tissues of } \mathrm{FH} n=6 \\
\text { samples per tissue }\end{array}$} & Capsule & 30 minutes & No detection \\
\hline & & Periarticular muscles & & \\
\hline & & Gluteus maximus muscle & & \\
\hline & & Round ligament & & \\
\hline & \multirow[t]{4}{*}{ Organs $n=6$ samples per organ } & Lung & 30 minutes and 24 hours & No detection \\
\hline & & Liver & & \\
\hline & & Spleen & & \\
\hline & & Kidney & & \\
\hline & \multirow{7}{*}{\multicolumn{2}{|c|}{ Blood $n=4$ samples per condition }} & 0 & No detection \\
\hline & & & 1 minute & \\
\hline & & & 5 minutes & \\
\hline & & & 15 minutes & \\
\hline & & & 30 minutes & \\
\hline & & & 1 hour & \\
\hline & & & 24 hours & \\
\hline & \multirow{2}{*}{\multicolumn{2}{|c|}{ Bone marrow $n=4$ samples per condition }} & 0 & No detection \\
\hline & & & 24 hours & \\
\hline
\end{tabular}

FH, femoral head; hBMSCs, human bone marrow mesenchymal stromal cells.

growth plate (Figure 3e). More precisely, dark cells were attached on the bone trabeculae (Figure 3i).

\section{Biodistribution of autologous pig bone marrow} mesenchymal stromal cells after intra-osseous injection in pig femoral head

To confirm our previous results, we wanted to use autologous pig cell grafting. BM from pig humerus was used to isolate pBMSCs and then these cells were characterized by their differentiation properties (Figure 4a-c). Porcine cells deposited an extensive mineralized matrix when cultured for 10 days in the osteogenic medium as demonstrated by strong Alizarin Red staining (Figure 4a). These porcine cells also efficiently differentiated into the adipogenic lineage, as indicated by Oil Red $\mathrm{O}$ staining of lipid droplets in the cytoplasm after 10 days of culture in an adipogenic medium (Figure 4b). The chondrogenic potential of pBMSCs was also highlighted by Alcian Blue staining after 3 weeks in a chondrogenic medium. This blue staining revealed a homogenous deposition of proteoglycan within the whole section of the pellet culture, and the counterstaining with hematoxylin showed the presence of chondrocyte-like cells in purple inside the lacunae (Figure 4c).

To be able to follow the autologous injected cells in the pig $\mathrm{FH}, \mathrm{pBMSCs}$ were stained with $\mathrm{DiOC}_{18}$ before injection. Compared with the unstained pBMSCs (control), indicated by dark histogram, the pBMSCs stained by $\mathrm{DiOC}_{18}$ (grey histogram) showed a 2 log switch of fluorescence, indicating that $100 \%$ of pBMSCs were positive for $\mathrm{DiOC}_{18}$ (Figure $4 \mathrm{~d}$ ). No positive cells were found in the different tissues and fluid tested in different times (blood and organs). Only normal tissue-specific cells were found (white histogram).

Bone tropism of bone marrow mesenchymal stromal cells To confirm that BMSCs have a preferential tropism for the bone surface, we assessed the capacity of the cells to locate and induce bone formation after a local delivery of the cells on a bone scaffold. For this purpose, an immunodeficient mouse model of ectopic implantation was performed. In the unseeded controls, no new bone formation was detected, and only scaffold, adipose tissue, 


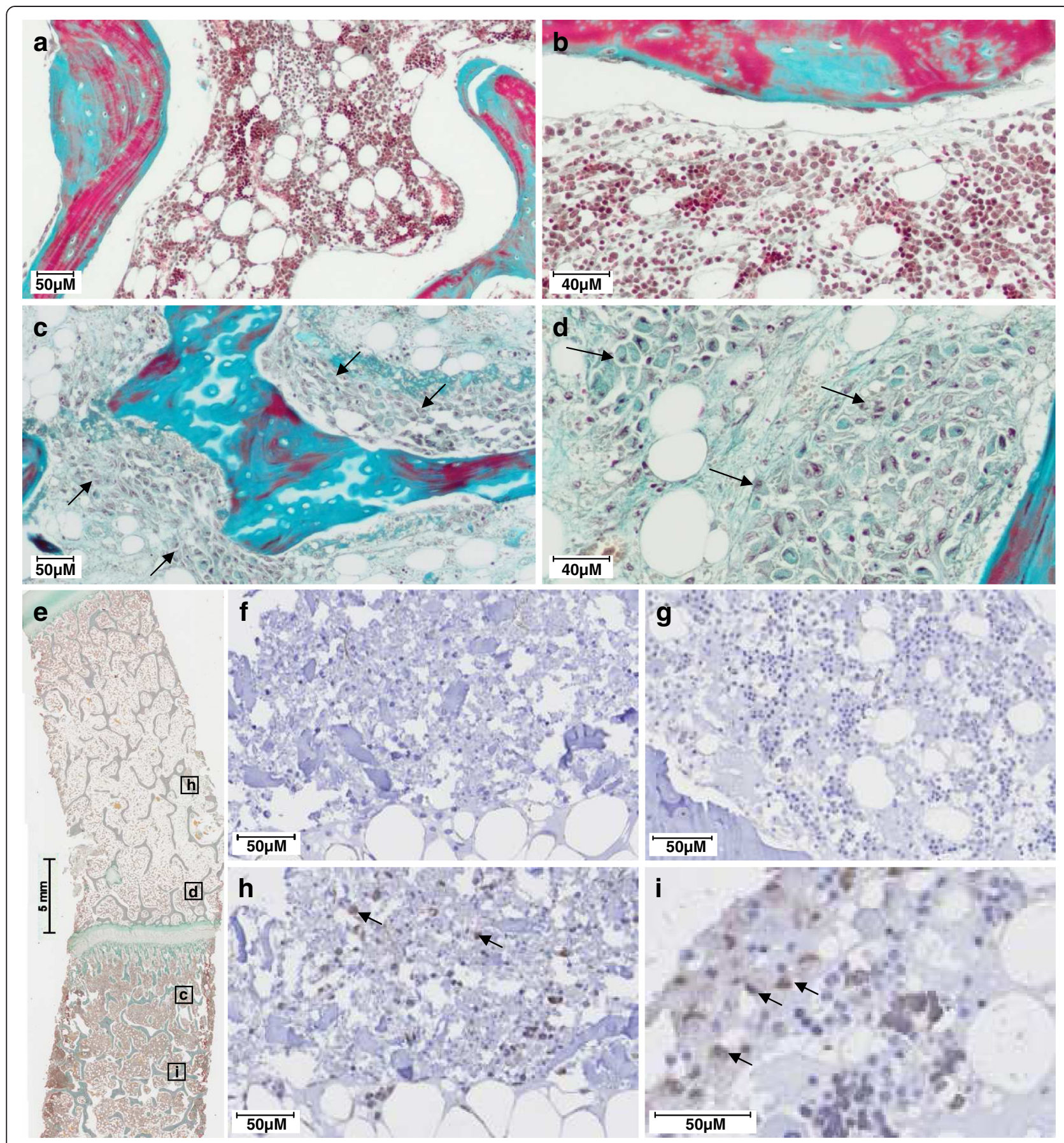

Figure 3 Detection of injected cells in pig femoral head (FH) 30 minutes after human bone marrow mesenchymal stromal cell (hBMSCs) injection. (a-e) Masson's Tri-chrome staining. (a, b) Normal pig FH sections. (c-e) Injected pig FH sections. Arrows indicate the hBMSC area. (e) Localization of sections (c), (d), (h), and (i). (f-i) In situ hybridization of human Alu sequences (Alu-ISH). (f) Negative control tissue of pig FH injected with human cells in omitting Alu probe stained with the hematoxylin only. (g) Negative control tissue of pig FH injected with physiological saline without human cells, with Alu probe and hematoxylin counterstaining. $(h, i)$ Injected pig FH with human cells detected by Alu-probe staining and counterstained with the hematoxylin. The positive nuclei for Alu-probe staining appear in dark brown (arrows). Magnifications: $10 \times(a, c, f-h), 20 \times(b, d), 1 \times(e)$, and $40 \times(i)$.

and loosely organized connective tissues were present (Figure 5a, b). In contrast, when pBMSCs were added onto the scaffold during the surgery, histological analysis revealed new bone formation with bone tissue containing osteocyte-like cells and osteoblast-like cell lining at the surface (Figure 5c, d). Altogether, this result 

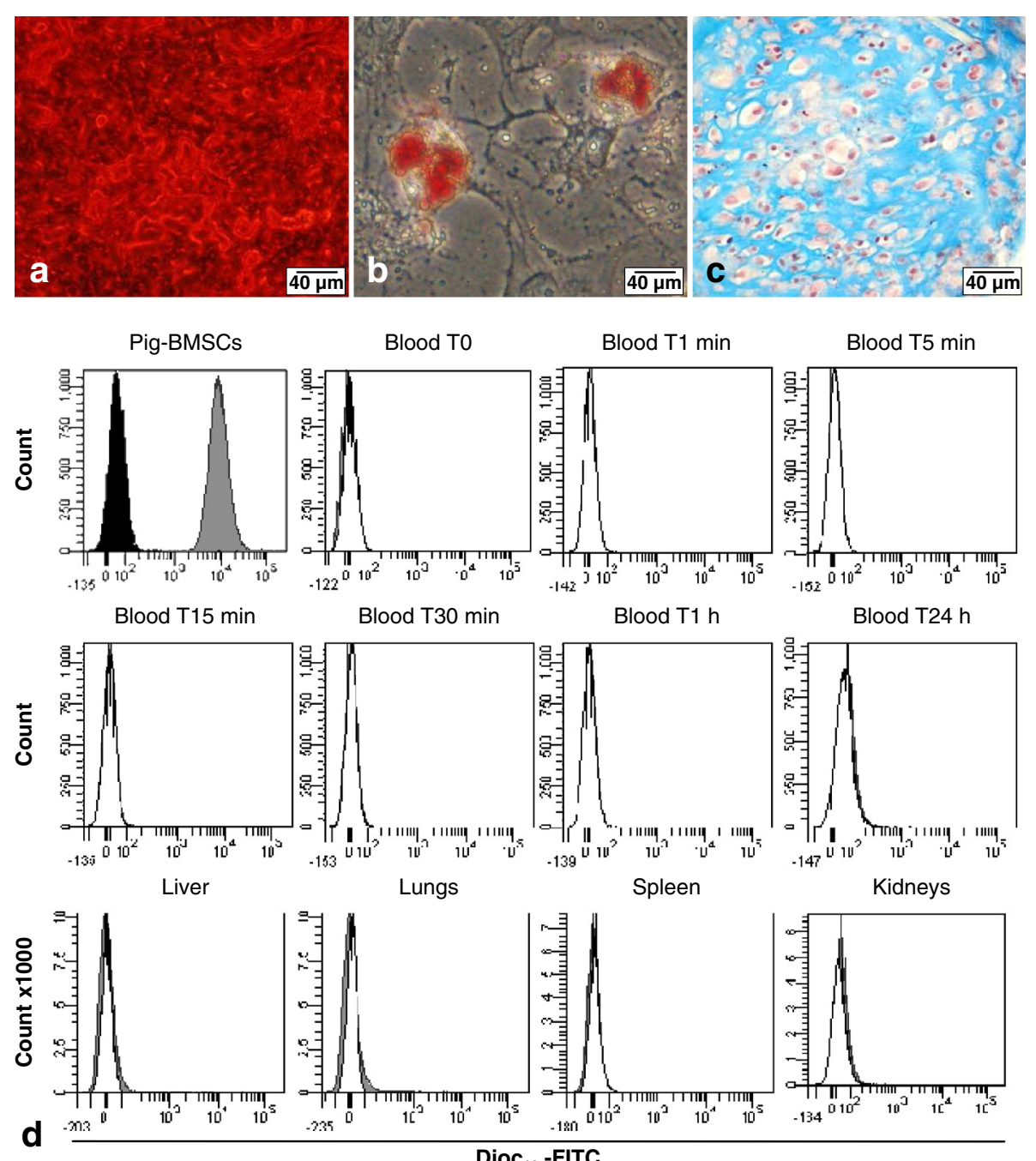

Figure 4 Biodistribution analysis of injected autologous pig bone marrow mesenchymal stromal cells (BMSCs) in pig femoral head by flow cytometry. Characterization of pig BMSCs in vitro with (a) osteoblastic differentiation with an extensive mineralized matrix of calcium hydroxyapatite crystals stained in red by Alizarin Red S, (b) adipocyte differentiation with adipocytes containing lipid droplets in the cytoplasm stained in red by Oil Red $\mathrm{O}$, and (c) chondrogenic differentiation with chondrocyte-like cells stained by hematoxylin in purple and surrounded by glycosaminoglycan-rich extracellular matrix stained in blue by Alcian Blue. (d) Biodistribution analysis by flow cytometry follow-up during 24 hours $(n=1)$ of autologous labeled DiOC 18 -FITC BMSCs injected in subchondral area of pig femoral head. Dark histogram: unstained pig BMSCs; grey histogram: stained DiOC 18 -FITC pig BMSCs; and white histogram: negative control cells of a non-injected pig. DiOC $18,3,3$ '-dioctadecyloxacarbocyanine perchlorate; FITC, fluorescein isothiocyanate.

indicated that pBMSCs preferentially located on bone surface and had the capacity to induce bone formation in vivo.

\section{Cell therapy of natural osteonecrosis of femoral head}

nONFH was diagnosed in pig. Compared with coronal MRI images of control pig FH (Figure 6a, f), nONFH was observed by a classic MRI appearance of osteonecrosis with a front regeneration: a geographical region of decreased marrow signal within the normally bright fat of the FH on T1-weighted images and mixed low/ high signal on T2 FS-weighted images (Figure 6b, g).
The front of regeneration delimitated a fatty necrotic area in the epiphysis of high T1 signal and a neutral signal on T2 FS-weighted images. One month after the first MRI, a new MRI analysis was conducted before a possible cell therapy. The diagnosis of osteonecrosis and the absence of spontaneous bone regeneration were confirmed by the observation of results similar to the previous analysis (Figure 6c, h).

After pBMSC injection in the center of nONFH, the pig was able to bear its full weight on the day of surgery. After 2 weeks, the front of regeneration had partially disappeared (Figure 6d, i). Nine weeks after pBMSC 

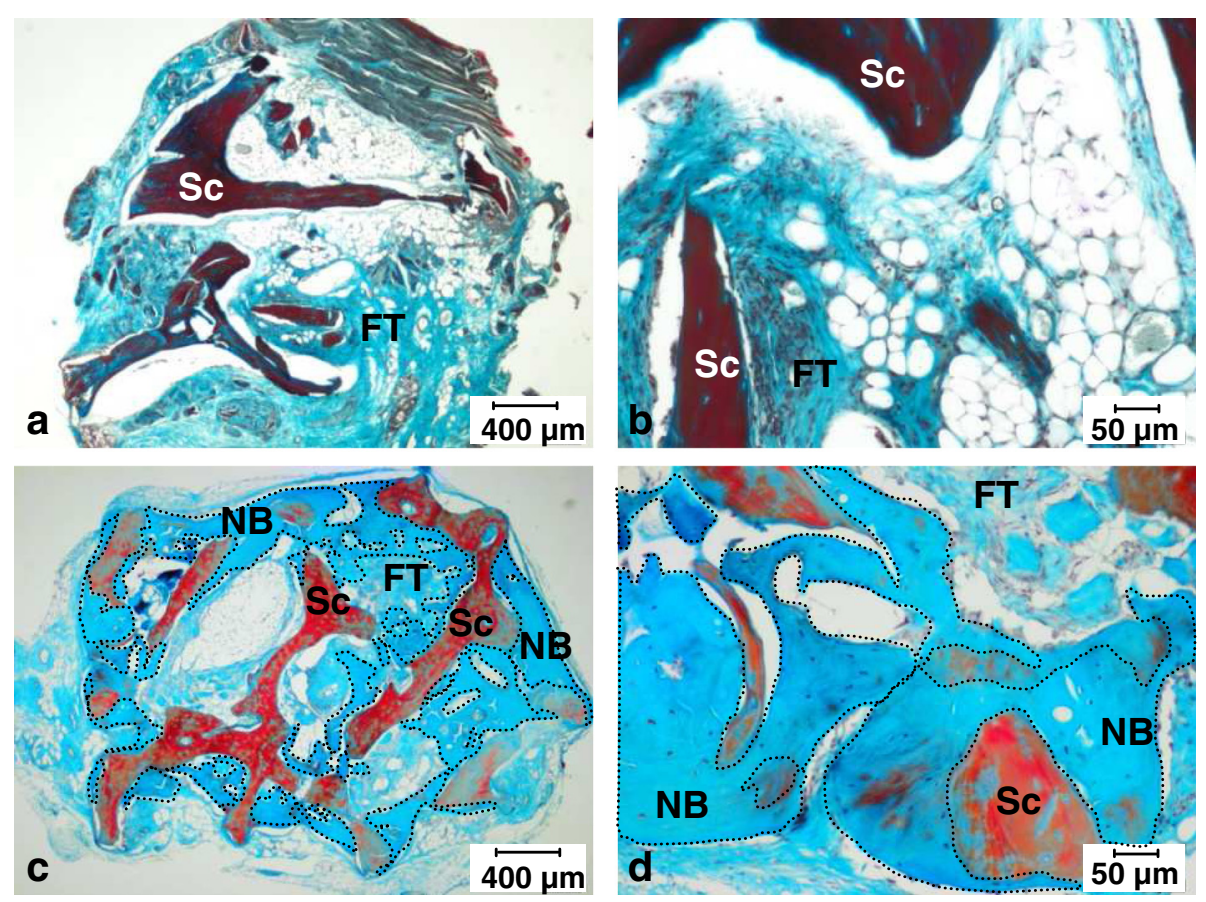

Figure 5 Histological analysis of in vivo bone formation after 7 weeks of ectopic implantation into immunodeficient mice. Scaffolds (a, b) without pig bone marrow mesenchymal stromal cells (pBMSCs) (a) Magnifications: 4x, (b) Magnifications: 20x. (c, d) with 300,000 pBMSCs directly loaded onto the bone scaffold during the surgery. Cells from three different pigs were tested. (c) Magnifications: $4 \times$, (d) Magnifications: 20x. Decalcified implants ( $n=6$ per condition) were embedded in paraffin and stained with Masson's Tri-chrome (blue/green = collagen and non-mineralized bone; red = mineralized bone scaffold; purple = nuclei; and pink = cytoplasm). Dotted lines correspond to the areas of new bone formation. NB, new bone; Sc, scaffold ; FT, fibrous tissue.

treatment, coronal T1 and T2 FS MRI showed no FH collapse and the front of regeneration was almost invisible. MRI FH images presented almost normal fatty bone medullary signal (Figure 6e, j). Then histological analysis was performed. Bone tissue showed a regular trabecular bone network with a normal growth plate in the middle of the FH (Figure 6k). The trabecular bones contained osteocytes and were surrounded by a normal BM constituted with hematopoietic cells and adipocytes (Figure 6l). No features of osteonecrosis such as osteocyte or adipocyte necrosis were visible. Only normal bone was observed.

\section{Discussion}

For ATMPs like hBMSCs, regulatory authorities require safety data concerning their innocuity and their biodistribution after their injection on a large animal model $[16,17]$. It has been previously shown that there is no risk of hBMSC transformation after their amplification and graft in vivo [18]. Although the safety and efficacy of BMSC injection have been tested mainly by using immunodeficient mice, we used the pig as a preclinical model, and for the first time we showed the biodistribution of cell injection by using either autologous pBMSCs or hBMSCs. Our results indicate that BMSCs remained confined at the site of injection and have the potential to repair the osteonecrosis in vivo. Currently, there is no single satisfactory method for assessing biodistribution. Several non-invasive, imaging-based monitoring methods have been used to track cell transplants via radiolabels, ferromagnetic particles (that is, iron oxide nanoparticles), and genetically modified cells engineered to express reporter transgenes but these approaches can functionally alter the cells [30-34]. To avoid alteration of BMSC functionality, hBMSCs were directly injected in the FH. Therefore, we used several highly sensitive techniques for cell detection, like flow cytometry, real-time qPCR, histology, and Alu-ISH analysis, in order to validate our results. qPCR is a quantitative and highly sensitive technique which allows the presence of a few human cells to be detected even when they are mixed with pig DNA. Our standard curve indicated that we were able to determine the exact number of human cells up to 13 human cells mixed in 3,200 pig cells. Below, human cells were detected but their number was not quantifiable. In the present study, our data demonstrated that after direct loading, grafted hBMSCs did not have unwanted homing. Indeed, no human cells were detected throughout the kinetic, from 30 minutes to 24 hours after implantation in tissues such as blood, BM, lungs, liver, spleen, and kidneys. Similarly, in tissues located near the drilling injection, such as capsule, round ligament, periarticular muscles, and gluteus maximus muscle, human cells were absent 

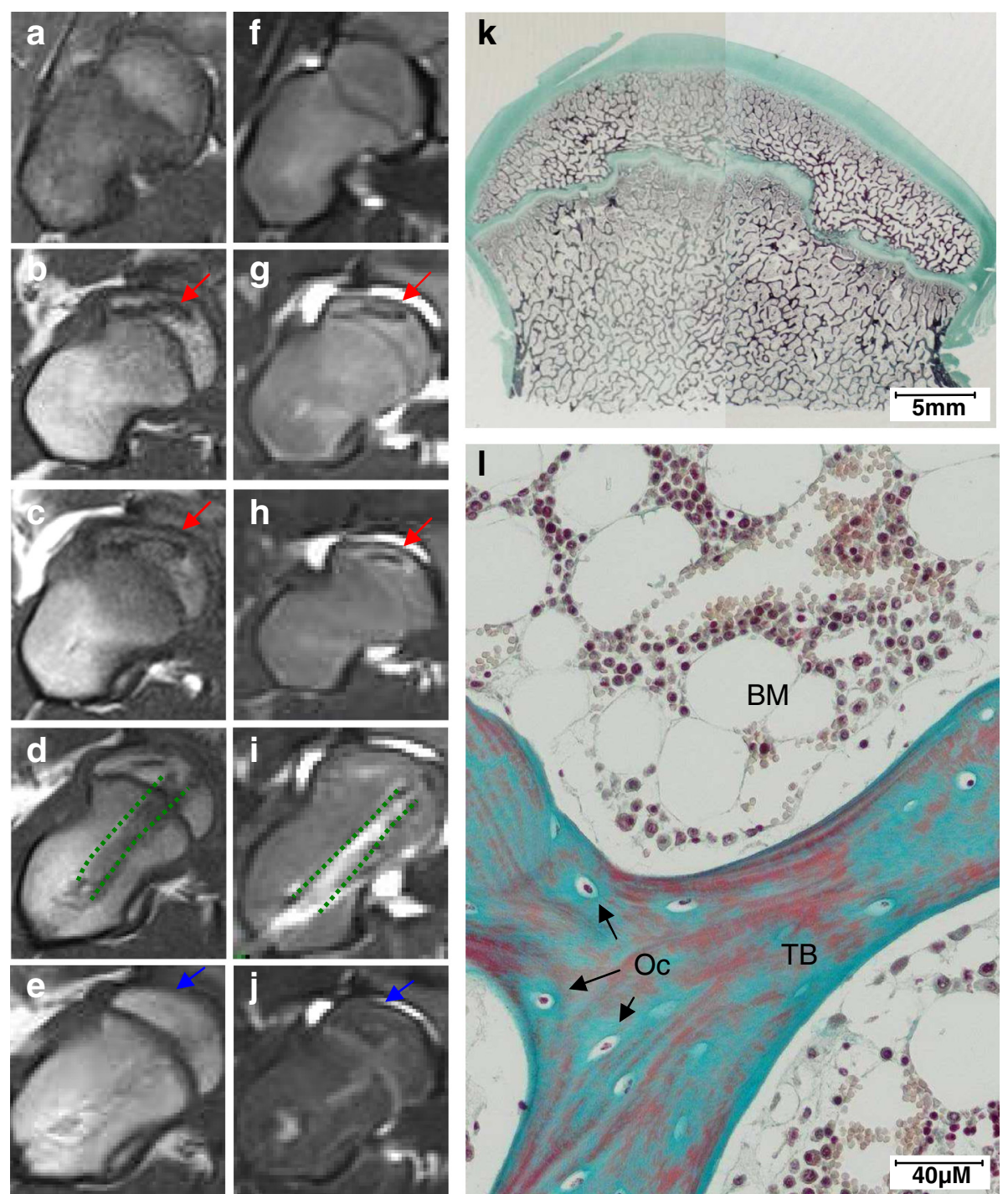

Figure 6 Cell therapy of natural femoral head osteonecrosis (nONFH) in the pig with injection of $140 \times 10^{6}$ autologous pig bone marrow mesenchymal stromal cells (pBMSCs) $(n=1)$ in femoral head $(F H)$. (a) to (j) Coronal magnetic resonance imaging (MRI) analysis: red arrows indicate necrotic area, green dashed lines indicate drilling rearrangement, and blue arrows indicate repair area. (a) to (e) T1-weighted image. (f) to (j) T2-FS (fat saturation)-weighted image. (a) and (f) Normal pig FH (negative control). (b) and (g) Diagnosis of nONFH in pig FH. (c) and (h) Confirmation of nONFH in pig FH 1 month after MRI diagnosis. (d) and (i) Pig FH with nONFH 2 weeks after injection of autologous pBMSCs. (e) and (j) Pig FH with nONFH 9 weeks after injection of autologous pBMSCs. (k) and (I) Histological analysis of pig FH with nONFH 9 weeks after injection of autologous pBMSCs stained with Masson's Tri-chrome (blue/green = collagen and non-mineralized bone; red = mineralized bone; purple $=$ nuclei). Magnifications: $1 \times(\mathbf{k})$ and $20 \times(\mathbf{I})$. BM, bone marrow; Oc, osteocytes; TB, trabecular bone.

at 30 minutes post-transplant. Human cells remained at the implantation site, in the $\mathrm{FH}, 30$ minutes after cell graft with an average of $147 \pm 21$ human cells per $10 \mathrm{mg}$ of $\mathrm{FH}$.

The drawback of this technique is the difficulty to clearly discriminate between viable BMSCs or macrophages which contain phagocytized human cell fragments [35]. To this end, qualitative analyses were performed. Because of their high repetition and species specificity, Alu sequences are a marker of choice to use ISH to detect few human cells in pig tissue [16]. This sensitive qualitative technique precisely localizes the cells in FH and indicates a good homogeneity of human cell distribution with the histological analysis. These results confirmed that grafted hBMSCs remained on trabeculae and provided evidence of the absence of spreading of these cells to the other tissues. Even if cells are described as immune-privileged with immunomodulatory, anti-inflammatory, immunosuppression potential and are used for xenogenic transplantation [36,37], we wanted to confirm our results by using autologous cell grafting. For this purpose, pig cells were stained with 
$\mathrm{DiOC}_{18}$, a green fluorescent membrane dye which does not induce adverse cellular effects [38]. The only drawback is that the cells can lose half of their fluorescence after doubling. However, human and pig BMSC doubling time is between 50 and 55 hours [7,39], and our followup does not exceed 24 hours. One day after pBMSC injection in the $\mathrm{FH}$, the flow cytometry analysis indicated that the injected cells were detected neither in blood nor in filter organs.

In contrast to studies in which cells are injected intravenously, our results confirm that injected cells preferentially stay in the injected tissue $[13,14,40,41]$. In our case, this can be explained by the fact that bone is the physiological niche of BMSCs. We confirmed their tropism for the bone surface with the ectopic bone implantation model in immunodeficient mice as only bone scaffold receiving pBMSCs during surgery gives bone formation. Moreover, our results with pBMSCs are in accordance with recent data we published showing that hBMSCs stay on the scaffold and do not migrate to other organs and this was the case throughout the 6 weeks of the study [42]. On the other hand, our data of grafted autologous pBMSCs in nONFH in the pig support that a local delivery of BMSCs into the FH during surgery facilitates their attachment and their participation in tissue regeneration. Altogether, these results indicate that a direct injection of BMSCs on the bone site is a safe procedure, even if a 4-mm diameter trocar is used to inject the cells into the FH. Clearly, these results indicate that there is no need to use a cell carrier like hydrogel or a plug after cell injection to obtain good cell localization without dissemination throughout the body. This is an important point as it was not possible to inject the cells into the FH when cells were mixed with hydrogel (data not shown). Finally, the feasibility of this approach is supported by the commonly used treatment of injection of concentrate $\mathrm{BM}$ in $\mathrm{FH}$ for the early stages of ONFH. Effectively, no side effects and even a positive effect have been observed in patients $[3,4,43]$.

Our preliminary data for the efficacy of grafted autologous BMSCs in $\mathrm{nONFH}$ in the pig seem promising and show a potential of BMSCs in the repair of necrosis in vivo. However, nONFH is rare in pigs. To have a statistically relevant result of the effectiveness of BMSCs on this painful disease, it is necessary to test this approach on one of the models of ONFH which has been developed in either emu or pig and to conduct a study on a large number of animals $[26,44]$. As the effectiveness of BMSCs has been proven and we and others showed the safety of this approach, the second strategy could be to go directly to the clinic $[6,7,13,14,18,40,41]$.

BMSC graft is a promising therapeutic approach for treating ONFH but the other question is the effectiveness of the BMSCs from patients with ONFH. Previous data from Yoo et al. described the good osteogenic abilities in vitro of BMSCs from patients with osteonecrosis [45]. Altogether, these data indicate that the use of autologous BMSCs for a therapeutic treatment of ONFH is a feasible strategy.

\section{Conclusions}

In summary, we have demonstrated in a large animal model that the intra-osseous portal is a safe and promising strategy for cell therapy treatment of FH osteonecrosis. As it has been previously shown that there is no risk of transformation of hBMSCs after their amplification and graft in vivo [18], this new study contributes to the preclinical data which are required for the clinical application of hBMSCs in the context of ATMPs.

\section{Abbreviations}

aMEM: alpha-modified Eagle's medium; Alu-ISH: in situ hybridization of human Alu sequences; ATMP: advanced therapy medicinal product; BM: bone marrow; BMSC: bone marrow mesenchymal stromal cell; Ct: cycle threshold; Dex: dexamethasone; DiOC 18: 3, 3'-dioctadecyloxacarbocyanine perchlorate; FCS: foetal calf serum; FH: femoral head; FS: fat saturation; gDNA: genomic deoxyribonucleic acid; hBMSC: human bone marrow mesenchymal stromal cell; ISH: in situ hybridization; IV: intravenous; MRI: magnetic resonance imaging; MSC: mesenchymal stromal cell; nONFH: natural osteonecrosis of femoral head; ONFH: osteonecrosis of the femoral head; pBMSC: pig bone marrow mesenchymal stromal cell; qPCR: quantitative real-time polymerase chain reaction; RT: room temperature; SCID: severe combined immunodeficiency; SSC: sodium saline citrate.

\section{Competing interests}

The authors declare that they have no competing interests.

\section{Authors' contributions}

AL carried out the BMSC culture and characterization, the biodistribution studies in flow cytometry, molecular biology, the ectopic implantation procedure, and all histological studies; performed data analysis and interpretation; and drafted the manuscript. AP carried out the surgical procedure in pigs and BM collection, participated in MRI analysis, drafted the surgical procedure part of the Methods section, and helped to revise the manuscript. MC performed the MRI studies and analyses and drafted the MRI part of the Methods and Results sections. JA carried out the study of ISH of human Alu sequences and drafted the part of Methods section corresponding to this procedure. $J$ participated in the biology molecular and flow cytometry studies and helped to revise the manuscript. PH conceived the injection procedure in $\mathrm{FH}$ and participated in the surgical procedure in pigs and in human BM collection. AR performed the MRI studies and analyses and helped to revise the manuscript. PB participated in the design of the study and helped to revise the manuscript. PL participated in the conception of the study and drafted the result section corresponding to the study of ISH of human Alu sequences. HR participated in conception and coordination of the study and in human BM collection and helped to revise the manuscript. NC designed and supervised the study, performed data analysis and interpretation, and drafted the manuscript. All authors read and approved the final manuscript.

\section{Acknowledgements}

This work was supported by the 7th Framework Program of the European Commission through the REBORNE (Regenerating BOne defects using New biomedical Engineering approaches) project (Health-2009-1.4.2-241879) and EFS Ile de France. We thank the Centre de Recherches Chirurgicales Dominique Chopin directed by René Yiou for the use of their animal platform facilities. The authors are grateful to Philippe Druelle and Philippe Mario for their invaluable help during surgery and animal care. We thank the histopathology of human and animal models service of the Pasteur Institute of Paris and give special thanks to Laurence Fiette. 


\section{Author details}

"Université Paris-Est, Faculté de médecine, Laboratoire de "Bioingénierie cellulaire, tissulaire et sanguine", EA3952, 5 rue Gustave Eiffel, 94000 Créteil, France. ¿Etablissement Français du Sang d'lle-de-France, Unité d'Ingénierie et de Thérapie Cellulaire, 5 rue Gustave Eiffel, 94017 Créteil cedex, France. ${ }^{3}$ Inserm UMR955, IMRB, 51 Avenue du Maréchal de Lattre de Tassigny, 94000 Créteil, France. ${ }^{4}$ Service de chirurgie orthopédique et traumatologie, AP-HP Hôpital Henri-Mondor, 51 Avenue du Maréchal de Lattre de Tassigny, 94000 Créteil, France. ${ }^{5}$ Service de radiologie Albert Chenevier, AP-HP Hôpital Henri-Mondor, 51 Avenue du Maréchal de Lattre de Tassigny, 94000 Créteil, France. ${ }^{6}$ Inserm U957, Laboratory for Pathophysiology of Bone Resorption, Faculty of Medicine, University of Nantes, 1 rue Gaston Veil, 44035 Nantes cedex 1, France. ${ }^{7}$ AP-HP Hôpital Henri-Mondor - A. Chenevier, Service hospitalier, 51 Avenue du Maréchal de Lattre de Tassigny, 94000 Créteil, France.

Received: 20 August 2014 Revised: 21 August 2014 Accepted: 4 March 2015 Published online: 13 April 2015

\section{References}

1. Levasseur R. Mechanisms of osteonecrosis. Joint Bone Spine. 2008;75:639-42.

2. Hungerford DS, Jones LC. Asymptomatic osteonecrosis: Should it be treated? Clin Orthop Relat Res. 2004;429:124-30.

3. Hernigou P, Beaujean F. Treatment of osteonecrosis with autologous bone marrow grafting. Clin Orthop Relat Res. 2002;405:14-23.

4. Gangji V, De Maertelaer V, Hauzeur JP. Autologous bone marrow cell implantation in the treatment of non-traumatic osteonecrosis of the femoral head: five year follow-up of a prospective controlled study. Bone. 2011:49:1005-9.

5. Wang X, Wang Y, Gou W, Lu Q, Peng J, Lu S. Role of mesenchymal stem cells in bone regeneration and fracture repair: A review. Int Orthop. 2013;37:2491-8

6. Petite $H$, Viateau V, Bensaid W, Meunier A, de Pollak C, Bourguignon M, et al. Tissue-engineered bone regeneration. Nat Biotechnol. 2000;18:959-63.

7. Chevallier N, Anagnostou F, Zilber S, Bodivit G, Maurin S, Barrault A, et al. Osteoblastic differentiation of human mesenchymal stem cells with platelet lysate. Biomaterials. 2010;31:270-8

8. Leotot J, Coquelin L, Bodivit G, Bierling P, Hernigou P, Rouard H, et al. Platelet lysate coating on scaffolds directly and indirectly enhances cell migration, improving bone and blood vessel formation. Acta Biomater. 2013;9:6630-40.

9. Quarto R, Mastrogiacomo M, Cancedda R, Kutepov SM, Mukhachev V, Lavroukov A, et al. Repair of large bone defects with the use of autologous bone marrow stromal cells. N Engl J Med. 2001;344:385-6.

10. Marcacci M, Kon E, Moukhachev V, Lavroukov A, Kutepov S, Quarto R, et al. Stem cells associated with macroporous bioceramics for long bone repair: 6- to 7-year outcome of a pilot clinical study. Tissue Eng. 2007;13:947-55.

11. Gao J, Dennis JE, Muzic RF, Lundberg M, Caplan Al. The dynamic in vivo distribution of bone marrow-derived mesenchymal stem cells after infusion. Cells Tissues Organs. 2001;169:12-20.

12. Lee RH, Pulin AA, Seo MJ, Kota DJ, Ylostalo J, Larson BL, et al. Intravenous hmscs improve myocardial infarction in mice because cells embolized in lung are activated to secrete the anti-inflammatory protein tsg-6. Cell Stem Cell. 2009;5:54-63.

13. Ramot $Y$, Meiron M, Toren A, Steiner M, Nyska A. Safety and biodistribution profile of placental-derived mesenchymal stromal cells (plx-pad) following intramuscular delivery. Toxicol Pathol. 2009:37:606-16.

14. Tolar J, O'Shaughnessy MJ, Panoskaltsis-Mortari A, McElmurry RT, Bell S, Riddle $\mathrm{M}$, et al. Host factors that impact the biodistribution and persistence of multipotent adult progenitor cells. Blood. 2006;107:4182-8.

15. Toupet $K$, Maumus M, Peyrafitte JA, Bourin P, van Lent PL, Ferreira $R$ et al. Long-term detection of human adipose-derived mesenchymal stem cells after intraarticular injection in scid mice. Arthritis Rheum. 2013;65:1786-94.

16. Sensebe L, Fleury-Cappellesso S. Biodistribution of mesenchymal stem/stromal cells in a preclinical setting. Stem Cells Int. 2013;2013:678063.

17. Sharpe ME, Morton D, Rossi A. Nonclinical safety strategies for stem cell therapies. Toxicol Appl Pharmacol. 2012;262:223-31.

18. Tarte K, Gaillard J, Lataillade JJ, Fouillard L, Becker M, Mossafa H, et al. Clinical-grade production of human mesenchymal stromal cells: Occurrence of aneuploidy without transformation. Blood. 2010;115:1549-53.
19. Breitbach M, Bostani T, Roell W, Xia Y, Dewald O, Nygren JM, et al. Potential risks of bone marrow cell transplantation into infarcted hearts. Blood. 2007;110:1362-9.

20. Zhu W, Xu W, Jiang $R$, Qian $H$, Chen $M, H u$ J, et al. Mesenchymal stem cells derived from bone marrow favor tumor cell growth in vivo. Exp Mol Pathol. 2006:80:267-74

21. Djouad F, Plence P, Bony C, Tropel P, Apparailly F, Sany J, et al. Immunosuppressive effect of mesenchymal stem cells favors tumor growth in allogeneic animals. Blood. 2003;102:3837-44.

22. Helke KL, Swindle MM. Animal models of toxicology testing: the role of pigs. Expert Opin Drug Metab Toxicol. 2013;9:127-39.

23. Merrifield CA, Lewis M, Claus SP, Beckonert OP, Dumas ME, Duncker S, et al. A metabolic system-wide characterisation of the pig: A model for human physiology. Mol Biosyst. 2011;7:2577-88.

24. Coquelin L, Fialaire-Legendre A, Roux S, Poignard A, Bierling P, Hernigou P, et al. In vivo and in vitro comparison of three different allografts vitalized with human mesenchymal stromal cells. Tissue Eng Part A. 2012;18:1921-31.

25. Bouderlique T, Henault E, Lebouvier A, Frescaline G, Bierling P, Rouard H, et al. Pleiotrophin commits human bone marrow mesenchymal stromal cells towards hypertrophy during chondrogenesis. PLoS One. 2014;9, e88287.

26. Poignard A, Lebouvier A, Cavet $\mathrm{M}$, Rahmouni A, Flouzat Lachaniette $\mathrm{CH}$ Bierling $P$, et al. New preclinical porcine model of femoral head osteonecrosis to test mesenchymal stromal cell efficiency in regenerative medicine. Int Orthop. 2014;38:1837-44.

27. Ozdemir O. Evaluation of human mast cell-mediated cytotoxicity by dioc18 target cell labeling in flow cytometry. J Immunol Methods. 2007;319:98-103.

28. Redwine JM, Armstrong RC. In vivo proliferation of oligodendrocyte progenitors expressing pdgfalphar during early remyelination. J Neurobiol. 1998:37:413-28.

29. Hernigou P, Homma Y, Flouzat Lachaniette CH, Poignard A, Allain J, Chevallier $\mathrm{N}$, et al. Benefits of small volume and small syringe for bone marrow aspirations of mesenchymal stem cells. Int Orthop. 2013;37:2279-87.

30. Bindslev L, Haack-Sorensen M, Bisgaard K, Kragh L, Mortensen S, Hesse B, et al. Labelling of human mesenchymal stem cells with indium-111 for spect imaging: Effect on cell proliferation and differentiation. Eur J Nucl Med Mol Imaging. 2006;33:1171-7.

31. Nohroudi K, Arnhold S, Berhorn T, Addicks K, Hoehn M, Himmelreich U. In vivo MRI stem cell tracking requires balancing of detection limit and cell viability. Cell Transplant. 2010;19:431-41.

32. Schmidtke-Schrezenmeier G, Urban M, Musyanovych A, Mailander V, Rojewski M, Fekete $\mathrm{N}$, et al. Labeling of mesenchymal stromal cells with iron oxide-poly(l-lactide) nanoparticles for magnetic resonance imaging: Uptake, persistence, effects on cellular function and magnetic resonance imaging properties. Cytotherapy. 2011;13:962-75.

33. Love Z, Wang F, Dennis J, Awadallah A, Salem N, Lin Y, et al. Imaging of mesenchymal stem cell transplant by bioluminescence and pet. J Nucl Med. 2007:48:2011-20.

34. Wang F, Dennis JE, Awadallah A, Solchaga LA, Molter J, Kuang Y, et al. Transcriptional profiling of human mesenchymal stem cells transduced with reporter genes for imaging. Physiol Genomics. 2009;37:23-34.

35. Warncke B, Valtink M, Weichel J, Engelmann $\mathrm{K}$, Schafer $\mathrm{H}$. Experimental rat model for therapeutic retinal pigment epithelium transplantation-unequivocal microscopic identification of human donor cells by in situ hybridisation of human-specific alu sequences. Virchows Arch. 2004;444:74-81.

36. Niemeyer P, Szalay K, Luginbuhl R, Sudkamp NP, Kasten P. Transplantation of human mesenchymal stem cells in a non-autogenous setting for bone regeneration in a rabbit critical-size defect model. Acta Biomater. 2010:6:900-8.

37. Maumus M, Guerit D, Toupet K, Jorgensen C, Noel D. Mesenchymal stem cell-based therapies in regenerative medicine: Applications in rheumatology. Stem Cell Res Ther. 2011;2:14.

38. Piriou L, Chilmonczyk S, Genetet N, Albina E. Design of a flow cytometric assay for the determination of natural killer and cytotoxic t-lymphocyte activity in human and in different animal species. Cytometry. 2000;41:289-97.

39. Peterbauer-Scherb A, van Griensven M, Meinl A, Gabriel C, Redl H, Wolbank S. Isolation of pig bone marrow mesenchymal stem cells suitable for one-step procedures in chondrogenic regeneration. J Tissue Eng Regen Med. 2010;4:485-90.

40. Detante O, Moisan A, Dimastromatteo J, Richard MJ, Riou L, Grillon E, et al. Intravenous administration of 99mtc-hmpao-labeled human mesenchymal 
stem cells after stroke: In vivo imaging and biodistribution. Cell Transplant. 2009;18:1369-79.

41. Allers C, Sierralta WD, Neubauer S, Rivera F, Minguell JJ, Conget PA.

Dynamic of distribution of human bone marrow-derived mesenchymal stem cells after transplantation into adult unconditioned mice. Transplantation. 2004; $78: 503-8$

42. Leotot J, Lebouvier A, Hernigou P, Bierling P, Rouard H, Chevallier N. Bone-forming capacity and biodistribution of bone marrow-derived stromal cells directly loaded into scaffolds: A novel and easy approach for clinical application of bone regeneration. Cell Transplant. 2014 Oct 28. [Epub ahead of print].

43. Gangji V, Hauzeur JP, Matos C, De Maertelaer V, Toungouz M, Lambermont M. Treatment of osteonecrosis of the femoral head with implantation of autologous bone-marrow cells. A pilot study. J Bone Joint Surg Am. 2004;86-A:1153-60.

44. Conzemius MG, Brown TD, Zhang Y, Robinson RA. A new animal model of femoral head osteonecrosis: One that progresses to human-like mechanical failure. J Orthop Res. 2002;20:303-9.

45. Yoo JJ, Song WS, Koo KH, Yoon KS, Kim HJ. Osteogenic abilities of bone marrow stromal cells are not defective in patients with osteonecrosis. In Orthop. 2009;33:867-72.

\section{Submit your next manuscript to BioMed Central and take full advantage of:}

- Convenient online submission

- Thorough peer review

- No space constraints or color figure charges

- Immediate publication on acceptance

- Inclusion in PubMed, CAS, Scopus and Google Scholar

- Research which is freely available for redistribution 